\title{
Sarcoma de Ewing extraóseo en la columna dorsal con compresión medular al momento del diagnóstico. Reporte de un caso
} Primary extra-osseous Ewing sarcoma of the dorsal spine with spinal cord compression at diagnosis. Case report

\author{
Dra. Gisel A. Bedoya $a^{a}$ Dra. Natali Bello ${ }^{a}$ Dra. Laura Monáa $a^{a}$ Dr. Kevin Velásqueza ${ }^{a}$ Dr. Jairo Sandoval ${ }^{b}$ \\ Dr. John F. Castro-Álvarez
}

\begin{abstract}
RESUMEN
Los tumores neuroectodérmicos primitivos a nivel extraóseo son una neoplasia muy rara, con pocas publicaciones en la literatura. Se reporta un caso de una paciente de nueve años quien se presentó con signos de compresión medular. Se estableció un grupo de diagnósticos diferenciales en el abordaje, entre los que debió incluirse la patología oncológica. En la paciente, se observó la presencia de una masa en la columna dorsal con compromiso del cordón medular, que requirió cirugía descompresiva de urgencia y, posteriormente, se diagnosticó sarcoma de Ewing. Cabe resaltar la importancia del caso debido a la baja incidencia del origen extraóseo en esta neoplasia y su rara localización en la columna dorsal, y destacar la presencia de la compresión medular como forma de presentación del cáncer en pediatría, además de la poca información que, hasta el momento, se posee referida al mejor abordaje terapéutico en esta patología, en esta localización.

Palabras clave: tumores neuroectodérmicos, columna vertebral, sarcoma de Ewing, neoplasias, niño.
\end{abstract}

\section{ABSTRACT}

Extraosseous primitive neuroectodermal tumors are very rare neoplasms. Only a few cases have been published in the literature. This report is about a 9-year-old female patient whose clinical manifestations showed spinal cord compression, so different diagnosis should be considered, including oncology disease. The patient showed a mass of the dorsal spine with spinal cord compression. She was diagnosed with Ewing Sarcoma after surgical intervention and pathology study. It is necessary to highlight the importance of the present case due to the low incidence of the extraosseous Ewing Sarcoma, and

a. Facultad de Ciencias de la Salud, Corporación Universitaria Remington, Medellín, Antioquia.

b. Hospital San Juan de Dios E. S. E. Rionegro, Antioquia.

c. Grupo de Investigación en Salud del Adulto Mayor, Facultad de Ciencias de la Salud, Corporación Universitaria Remington, Medellín.

Colombia.

Correspondencia:

Dr. John F. Castro-Álvarez: john.castro@uniremington.edu.co

Financiamiento: Ninguno.

Conflicto de intereses: Ninguno que declarar.

Recibido: 21-12-2017

Aceptado: 15-8-2018 its infrequent location at the dorsal spine and the spinal cord compression as initial presentation of pediatric cancer, as well as the poor information related to the best therapeutic strategy to treat this disease at this location.

Key words: neuroectodermal tumors, spine, sarcoma, Ewing, neoplasms, child.

http: / / dx.doi.org/10.5546/ aap.2019.e41

Cómo citar: Bedoya GA, Bello N, Moná L, Velásquez K, et al. Sarcoma de Ewing extraóseo en la columna dorsal con compresión medular al momento del diagnóstico. Reporte de un caso. Arch Argent Pediatr 2019;117(1):e41-e46.

\section{INTRODUCCIÓN}

Los tumores neuroectodérmicos primitivos (primitive neuroectodermal tumors; PNET, por sus siglas en inglés) son una enfermedad maligna infrecuente, de células pequeñas y redondas. ${ }^{1,2}$ Estos afectan huesos y tejidos blandos, principalmente en la región torácica, el retroperitoneo, la región paravertebral, la región de la cabeza, el cuello, y las extremidades. ${ }^{3}$ Los PNET se consideran tumores diferenciados cuando expresan marcadores neurales, mientras que el sarcoma de Ewing (SE), una lesión neoplásica perteneciente a la misma familia, siempre se considera indiferenciado, ya que no presenta compromiso neural. Al ser de la misma familia, ambos comparten el compromiso del cromosoma 22, con una translocación cromosómica característica; la más frecuente (en el $85 \%$ de los casos) es la (t11;22)(q24;q12), $\mathrm{y}$, en el $10 \%$ de los casos, la (t21;22)(q22;q12), que generan una proteína oncogénica de fusión EWSR1-FLI1, EWSR1-ERG, respectivamente, por lo que se consideran, en la actualidad, variantes del mismo tumor. ${ }^{3,4}$

El SE / PNET afecta, sobre todo, a niños y adolescentes, y es más común en los individuos de raza blanca y en el sexo masculino. ${ }^{5}$ Los síntomas y signos clínicos en esta localización son dolor, masa palpable y, dependiendo de la localización, asociarse a déficit neurológico. ${ }^{6}$ 
Puede presentar metástasis en un $25 \%$ de los casos al pulmón, al hueso, a la médula ósea y a los ganglios linfáticos. ${ }^{7}$ Radiológicamente, se asocia con una reacción perióstica en láminas o "piel de cebolla" ${ }^{5,7}$ La lesión es, por lo general, lítica y central con bordes, más o menos mal definidos o geográficos. ${ }^{5}$ También se puede presentar como una lesión esclerótica con expansión ósea. ${ }^{5}$

El objetivo de este trabajo es reportar el caso de una paciente femenina de nueve años a quien se le diagnosticó SE de origen extraóseo en la columna dorsal, con compresión medular al momento del diagnóstico, y los diferentes enfoques diagnósticos y terapéuticos utilizados.

\section{REPORTE DE CASO}

Paciente femenino de nueve años de edad quien presentó dolor progresivo a nivel interescapular con predominio en decúbito supino, con tiempo de evolución de 15 días al momento de su ingreso a Urgencias. Paciente con dificultad para la deambulación, dolor en la columna dorsal, de mayor intensidad en las horas de la noche, parestesias, pérdida de la fuerza en los miembros inferiores con predominio en el miembro inferior derecho, con antecedente de dos consultas recientes en las que se había diagnosticado un espasmo muscular y dolores del crecimiento.
Al momento del examen físico, se manifestó con dolor ante la palpación en la región dorsal interescapular, sin signos inflamatorios; no se palparon masas ni espasmos paravertebrales, arcos de movilidad de la cadera conservados, dificultad para la marcha, pérdida de la fuerza muscular en los miembros inferiores, más acentuada en el derecho, reflejos osteotendinosos disminuidos, simétricos y sensibilidad disminuida. En la radiografía de columna, se evidenció escoliosis dorsolumbar de convexidad izquierda, sin otros hallazgos. Al cabo de 12 horas, la paciente presentó deterioro del cuadro clínico con progresión del déficit motor y pérdida de la sensibilidad en los miembros inferiores. Se decidió trasladarla a la Unidad de Cuidados Intensivos para el monitoreo continuo y realizar una resonancia magnética nuclear ( $\mathrm{RMN})$ simple y contrastada de la columna total, y la interconsulta con Neurología por el presunto diagnóstico de Guillain-Barre.

La RMN reportó un colapso vertebral de T2 en aproximadamente un $50 \%$ asociado a una lesión de tejidos blandos que comprometía el espacio epidural con compresión del cordón medular y se extendía en sentido superior, inferior y forámenes (Figura 1). Entre las posibilidades diagnósticas, se consideró histiocitosis, linfoma y SE/PNET. Se interconsultó con Neurología Pediátrica, quienes

FIgURA 1. Resonancia magnética nuclear con dos cortes sagitales ( $A-B)$ y un corte coronal (C)
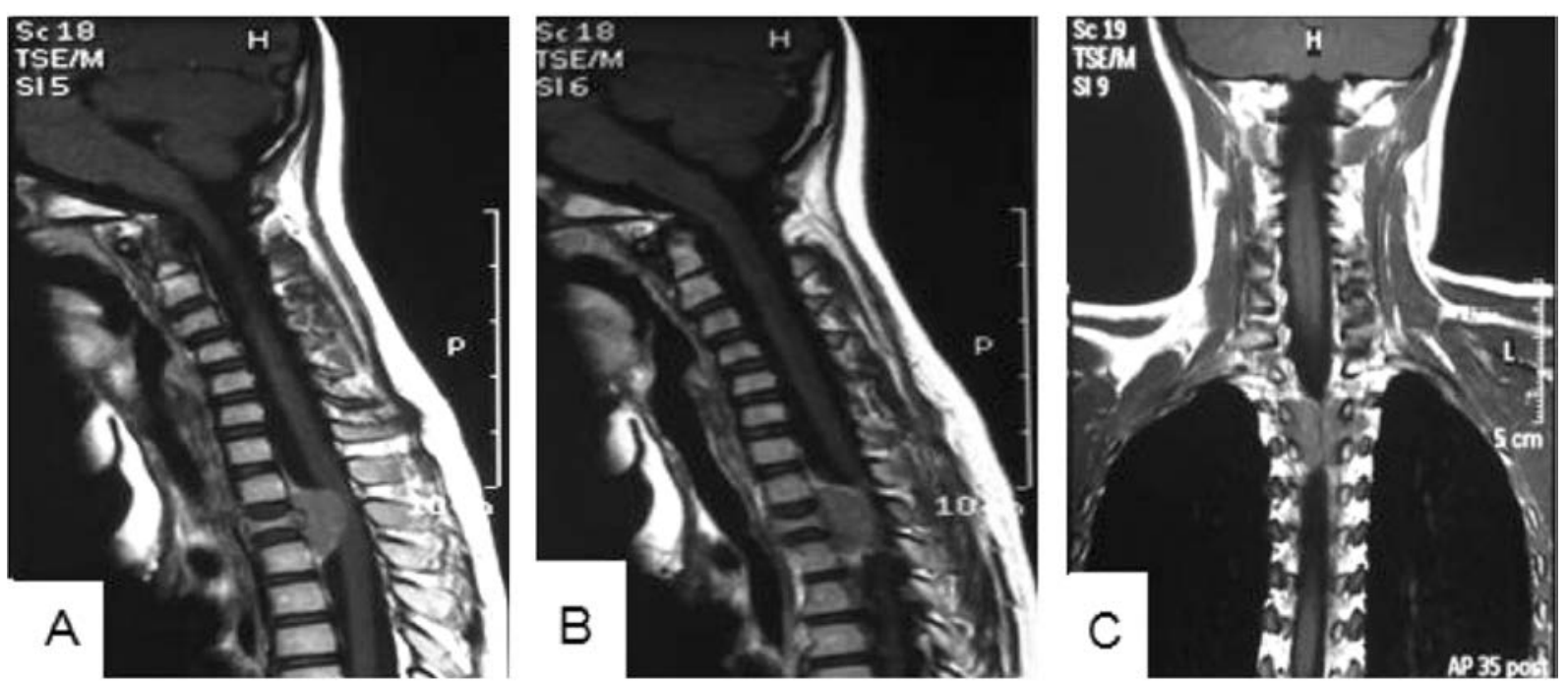

Las tres imágenes con modalidad T1 y preponderancia de la columna vertebral. En las figuras A y B, se aprecia la existencia de una masa hipointensa que hace compresión medular en C7-T1. En la imagen C, se observa la presencia de un cuerpo hipointenso en C7-T1 y T2. 
hicieron un diagnóstico de tumor extradural con compresión en el canal medular y sugirieron realizar la biopsia y la resección del tumor espinal.

Se realizó laminectomía total de T2 y parcial de T1 y T3 por el canal estrecho dorsal más la resección del tumor extraaxial dorsal, el cual era de características violáceas y muy vascularizado. Se resecó, se liberó el canal y se dejó la médula y las raíces nerviosas libres. En el reporte de la biopsia, se reveló un tumor extradural con fragmentos de una neoplasia maligna constituida por células pequeñas, redondas y azules con cromatina fina. Las células se teñían positivamente con el marcador CD99 y fueron negativas para CD45, CD20, TdT, sinaptofisina, miogenina y desmina, lo que confirmó un SE/ PNET (Figura 2).

A los quince días del posoperatorio, la paciente presentó deterioro motor y dolor dorsal, paraparesia espástica e hiperreflexia en los miembros inferiores. Se le realizó una tomografía axial computarizada (TAC) de control, que

FIGURA 2. Cortes tomados del tumor extradural que muestran fragmentos de una neoplasia maligna constituida por células de tamaño pequeño con cromatina fina
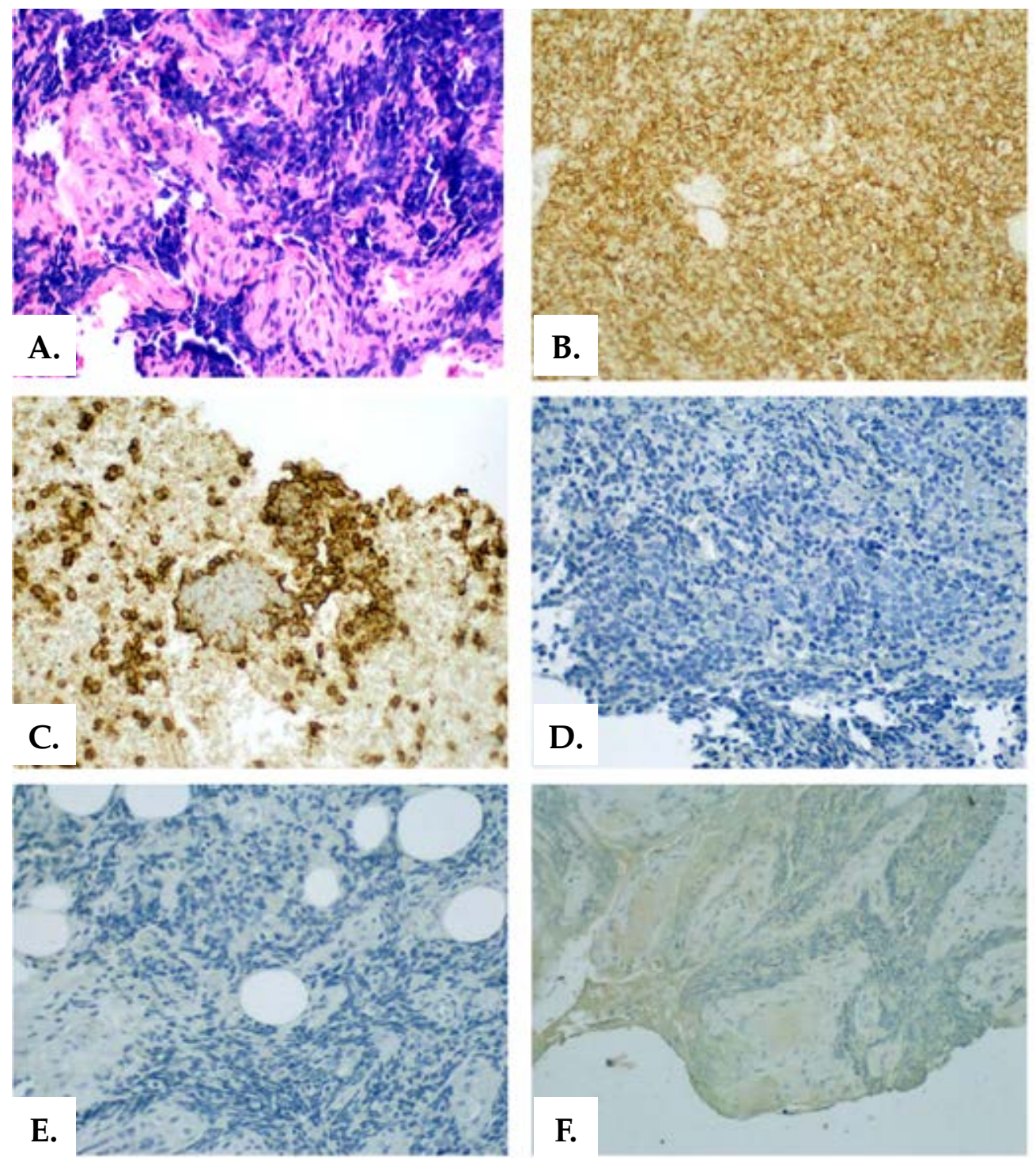

A. Hematoxilina y eosina (40X). B y C. CD99 positivo (40X). D. Sinaptofisina negativa (40X). E. Miogenina negativa. F. TdT negativa (40X). 
mostró una masa paravertebral derecha e izquierda que se extendía cranealmente al disco C7-T1 alrededor de ocho milímetros y caudalmente al cuerpo vertebral T2, obliteraba el flujo del líquido cefalorraquídeo, lo que comprometía la medula hasta en un $80 \%$. También se encontró un defecto de los elementos posteriores en este nivel y se observó la extensión a través de los forámenes de emergencia, por lo que fue evaluada por Oncología Pediátrica y se decidió iniciar quimioterapia y radioterapia. Se le realizó una gammagrafía ósea corporal total, que arrojó como resultado el estudio negativo para lesiones metastásicas. Luego de un año en tratamiento, se dio una recuperación de la movilidad y de la sensibilidad en los miembros inferiores y la ausencia de masas en la columna vertebral, lo que dio por culminado el tratamiento.

En la TAC de control realizada a los dos años del tratamiento, se observó, en el lóbulo inferior derecho del pulmón, un nódulo en el segmento lateral y un nódulo en el segmento anterior sugestivo de metástasis (Figura 3). Se le realizó una lobectomía segmentaria del lóbulo inferior y medio derecho, cuyo diagnóstico histológico fue positivo para metástasis de SE, y se inició el tratamiento de cuidados paliativos sin tratamiento quimioterapéutico.

Dos meses después de la recaída, la paciente presentó episodios de tos acompañados de dolor pleurítico en el hemitórax izquierdo. Se solicitó una radiografía de tórax, que evidenció un derrame pleural izquierdo masivo. Se realizó una toracocentesis evacuante, que fue poco efectiva, ya que se observó compromiso pleural por el tumor, y la paciente quedó con oxígeno suplementario de forma permanente. Se procedió a la realización de estudios complementarios, entre ellos, una gammagrafía ósea total, en la que se evidenció metástasis en el fémur, la pelvis, la parrilla costal y el húmero (Figura 4). Se decidió tenerla bajo sedación debido a su compromiso hemodinámico e inestabilidad del patrón respiratorio, con su posterior fallecimiento.

\section{DISCUSIÓN}

El SE es un tumor que posee origen primario, generalmente, a nivel óseo, pero puede tener un origen distinto desde estructuras blandas, tales como el músculo, el tejido adiposo, y, en algunos casos, desde estructuras serosas, como las adventicias o las meninges. ${ }^{8} \mathrm{La}$ incidencia general del SE lo ubica en el segundo tumor óseo en frecuencia, con distribución en los huesos largos, y en el esqueleto axial, alrededor del $55 \%$ y del $45 \%$, respectivamente, y es de origen extraóseo en acerca del 16-18 \% de los casos. ${ }^{9} \mathrm{El}$ origen tumoral extraóseo es infrecuente, como se describió en una serie de 1631 casos, en la que se reportaron 290 casos de localización extraósea $(18,4 \%)$ del SE. ${ }^{10}$ Por su parte, los SE epidurales extraóseos son extremadamente raros, con tan solo 32 casos reportados en la literatura en 2017. ${ }^{11}$ Los SE extraóseos vs. los óseos serían más frecuentemente axiales extrapélvicos, menores de 8 centímetros sin alteraciones genómicas diferentes entre los grupos. ${ }^{9}$

Figura 3. Tomografía axial computarizada con ventana al pulmón, en la que se observa la presencia de múltiples masas en el lóbulo inferior $(A)$ y medio $(B)$ del pulmón derecho

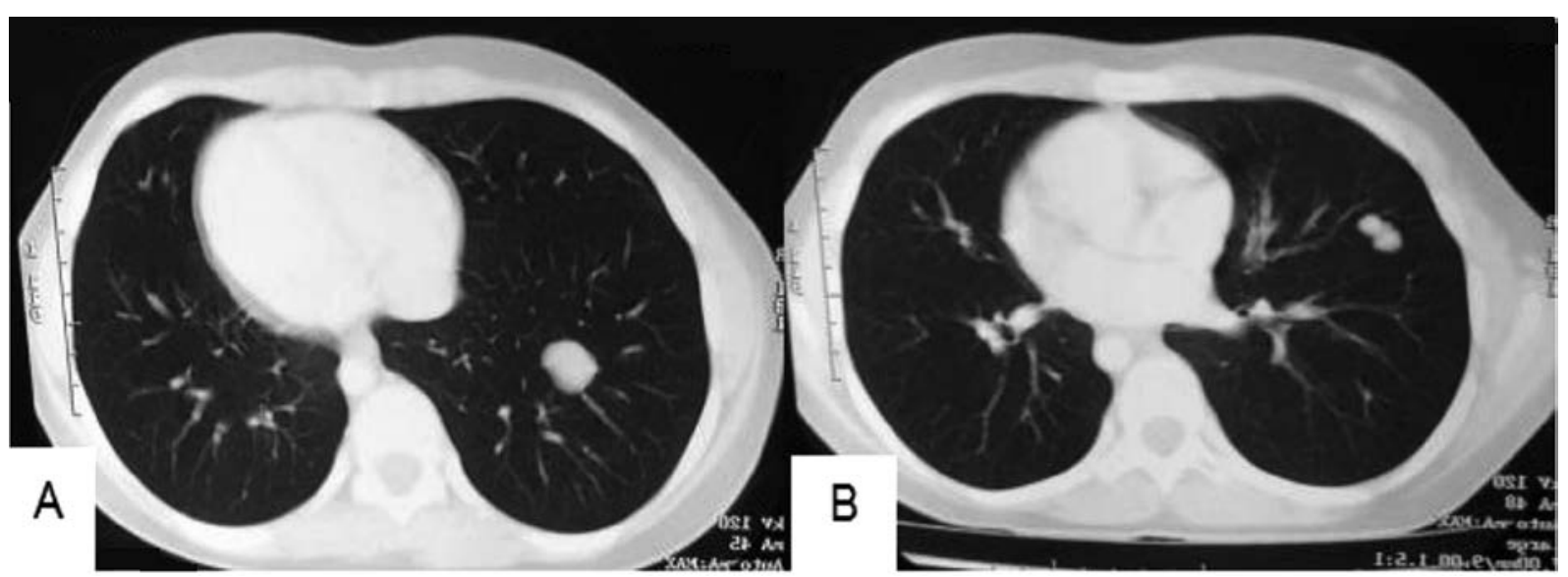

Estas masas tienen bordes regulares, definidos y asociados a la presencia de lesiones satélite y adenopatías. Las imágenes se encuentran invertidas, por artefacto de su escaneo. 
El caso clínico descrito da cuenta de una masa en la región dorsal de la columna vertebral, con origen extraóseo, cuyas características histológicas lo denominan como SE, con una localización poco frecuente en estas neoplasias. Se evidencia también un curso clínico larvado y lento, que no es habitual en las patologías de ortopedia oncológica. Sin embargo, presenta una recaída metastásica pulmonar y ósea, lo que le confiere muy mal pronóstico y le ocasiona el óbito a los tres meses de la recaída. Un 30-40\% de los pacientes con SE presentan recaída, y es aún más frecuente si el SE es metastásico al momento del diagnóstico, por lo general, en los dos primeros años, y la supervivencia para los pacientes con SE recaído es muy pobre, de, aproximadamente, el $10-15 \%$ a 5 años. ${ }^{4,12,13}$ Se han encontrado pocas publicaciones acerca del SE en la columna, que avalan el origen vertebral como prácticamente el único sitio de origen, y, en solo unos pocos casos, en las meninges perimedulares. ${ }^{8,14}$

No obstante, hay un reporte de cuatro casos de jóvenes que desarrollaron SE/PNET en la bóveda craneal, específicamente, a nivel supratentorial y en la fosa posterior ${ }^{8}$ mientras que, en este reporte de caso, aunque no fue posible identificar el origen de la lesión primaria, se tiene certeza de que estuvo comprometido el canal medular y las meninges. También refleja la rareza de esta patología la reciente publicación con 32 casos reportados de SE epidurales extraóseos en la literatura. ${ }^{11}$ Queda, finalmente, un panorama abierto para la investigación, la búsqueda y el reporte de más casos para poder entender el comportamiento genético, clínico e histopatológico de la enfermedad y la mejor modalidad terapéutica para utilizar.

\section{Agradecimientos}

A la Clínica Las Américas por el acceso a los hallazgos histológicos.

\section{REFERENCIAS}

1. Zhao M, Zhang B, Liang F, Zhang J. Primary spinal intradural extraskeletal Ewing sarcoma mimicking a giant nerve sheath tumor: Case report and review of the literature. Int J Clin Exp Pathol. 2014; 7(12):9081-5.

2. Mardekian S, Gandhe A, Miettinen M, Pack S, et al. Two Cases of Spinal, Extraosseous, Intradural Ewing's sarcoma/ Peripheral Neuroectodermal Tumor: Radiologic, Pathologic, and Molecular Analysis. J Clin Imaging Sci. 2014; 4:6.

3. Goldblum JR, Folpe AL, Weiss SW, eds. Enzinger and Weiss's soft tissue tumors. 6. ${ }^{\text {ta }}$ ed. Filadelfia, PA: Elsevier; 2014.

4. Gaspar N, Hawkins DS, Dirksen U, Lewis IJ, et al. Ewing Sarcoma: Current Management and Future Approaches Through Collaboration. J Clin Oncol. 2015; 33(27):3036-46.

5. Brenes Zuñiga MG. Sarcoma De Ewing: enfoque radiográfico. Revista Médica de Costa Rica y Centroamérica. 2014; 71(610):271-3.

6. De Ioris MA, Prete A, Cozza R, Podda M, et al. Ewing Sarcoma of the Bone in Children under 6 Years of Age. PLoS One. 2013; 8(1):e53223.

7. Downing JR, Head DR, Parham DM, Douglass EC, et al. Detection of the $(11 ; 22)(q 24 ; q 12)$ translocation of Ewing's sarcoma and peripheral neuroectodermal tumor by reverse transcription polymerase chain reaction. Am J Pathol.1993; 143(5):1294-300.

8. Ke C, Duan Q, Yang H, Zhu F, et al. Meningeal Ewing Sarcoma/Peripheral PNET: Clinicopathological, Immunohistochemical and FISH study of four cases. Neuropathology. 2017; 37(1):35-44.

9. Cash T, McIlvaine E, Krailo MD, Lessnick S, et al. Comparison of clinical features and outcomes in patients with extraskeletal versus skeletal localized Ewing sarcoma:

Figura 4. (A) Resonancia magnética nuclear contrastada de los miembros inferiores con múltiples focos metastásicos en los huesos largos. (B) Gammagrafía ósea en la que se aprecia la captación de las múltiples lesiones metastásicas

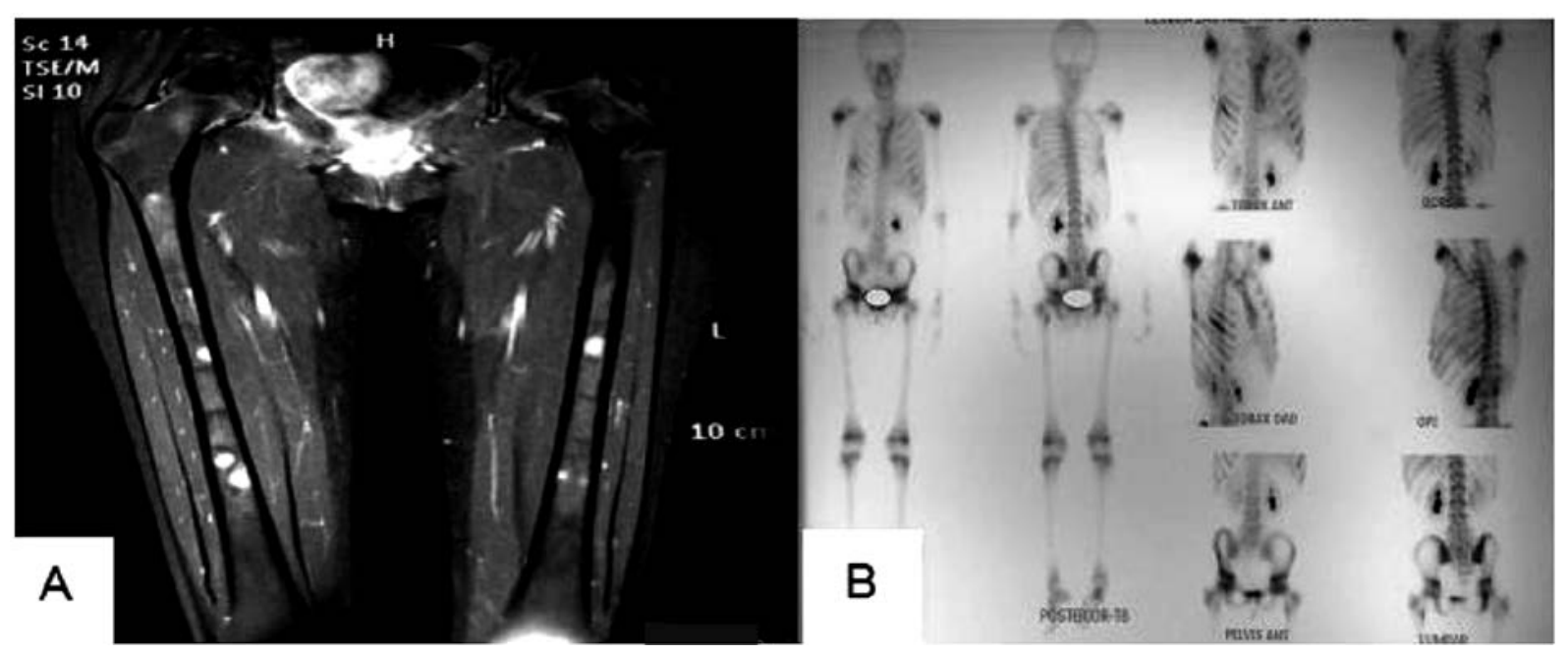


A report from the Children's Oncology Group. Pediatr Blood Cancer. 2016; 63(10):1771-9.

10. Jawad MU, Cheung MC, Min ES, Schneiderbauer MM, et al. Ewing sarcoma demonstrates racial disparities in incidence-related and sex-related differences in outcome: An analysis of 1631 cases from the SEER database, 19732005. Cancer. 2009; 115(15):3526-36.

11. Gopinath S, Moorthy P, Kulasekaran N. Pediatric Epidural Extra-Osseous Ewing's Sarcoma - Extremely Rare Case Report and Review of the Literature. Int J Radiol Radiat Ther. 2017; 4(3): 00096.
12. Ferrari S, Luksch R, Hall KS, Fagioli F, et al. Post-relapse survival in patients with Ewing sarcoma. Pediatr Blood Cancer. 2015; 62(6):9949.

13. Stahl M, Ranft A, Paulussen M, Bölling T, et al. Risk of recurrence and survival after relapse in patients with Ewing sarcoma. Pediatr Blood Cancer. 2011; 57(4):549-53.

14. Sharma C, Nath K, Kumawat BL, Acharya M, et al. Paraspinal extraskeletal Ewing's sarcoma: a rare clinical entity. BMJ Case Rep. 2014; 2014:bcr2014205010. 